\title{
Cervical Stenosis in Adult Arthrogryposis: A Case Report and Review of the Literature
}

\author{
Allen L. Ho ${ }^{1}$ Jyodi Mohole ${ }^{1} \quad$ Eric S. Sussman ${ }^{1}$ Arjun V. Pendharkar ${ }^{1}$ Harminder Singh ${ }^{1}$ \\ ${ }^{1}$ Department of Neurosurgery, Stanford University, Stanford, \\ California, United States

\begin{abstract}
Address for correspondence Harminder Singh, MD, Department of Neurosurgery, Stanford Hospitals and Clinics, 300 Pasteur Drive, Stanford, CA 94305, United States (e-mail: Harmansingh.md@gmail.com).
\end{abstract}

J Neurol Surg Rep 2018;79:e19-e22.

\begin{abstract}
Keywords

- Arthrogryposis

- cervical stenosis

- cervical fusion

- congenital disorders

- spinal deformity

Arthrogryposis multiplex congenita is a rare, nonprogressive congenital disorder that describes a constellation of conditions characterized by multiple joint contractures. Spinal pathology and deformity are common; however, the majority of the literature on arthrogryposis is focused on pediatric management. There exist a very few reports on long-term outcomes and management of adults with arthrogryposis. We present a case of cervical spinal stenosis in an adult female with arthrogryposis that underwent posterior cervical decompression and fusion. A review of spine-related sequelae seen in adults with arthrogryposis and considerations for spinal surgery for these patients is discussed.
\end{abstract}

\section{Introduction}

Arthrogryposis multiplex congenita (AMC) is a rare, nonprogressive congenital disorder that describes a constellation of conditions characterized by multiple joint contractures. ${ }^{1-3}$ The etiology of these contractures can be attributed to neurologic, muscular, genetic, or connective tissue abnormalities, all of which lead to impaired fetal movement and permanent contraction of muscle fibers. ${ }^{4,5}$ Because these deformities are present at birth, treatment begins at an early age. Therefore, the majority of the literature on arthrogryposis is focused on pediatric management. ${ }^{6}$ There exist very few reports on long-term outcomes and management of adults with arthrogryposis. ${ }^{3,6}$ Our report aims to contribute to this growing body of literature by presenting a case of cervical spinal stenosis in an adult female with arthrogryposis. A review of spine-related sequelae seen in adults with arthrogryposis and considerations for spinal surgery for these patients is also discussed.

\section{Case}

A 33-year-old female with a history of arthrogryposis presented to the emergency department in a wheelchair with a chief complaint of weakness and paresthesia in the right upper extremity. Onset of symptoms was 2 to 3 weeks prior to presentation, but progressive worsening was noticed in the last 5 days. At baseline, the patient used a walker for ambulation, but due to worsening upper extremity strength, she was now limited to using a wheelchair. Physical examination revealed motor weakness in all right upper limb muscle groups ( $4-/ 5$ deltoids, $4 / 5$ biceps, $4+/ 5$ triceps, $4 / 5$ wrist extensors, $4 / 5$ finger flexors). She reported paresthesias in her right hand and forearm. Hoffman's reflex was elicited bilaterally. Motor strength and sensation in the left upper limb and bilateral lower limbs were normal and all cranial nerves were intact. A comprehensive review of systems was otherwise unremarkable.

The patient had a long-standing history of arthrogryposis and underwent several lower extremity reconstructive surgeries from birth until 5 years of age. (-Fig. 1) Previous surgical history also included a T3 to L5 thoracolumbar fusion for scoliosis treatment as an adolescent. Other notable medical conditions included diabetes mellitus.

Magnetic resonance imaging (MRI) of the cervical spine revealed moderate-to-severe spinal stenosis from the $\mathrm{C} 3$ to C5 levels. (-Fig. 2) There was evidence of intramedullary spinal cord signal change at the $\mathrm{C} 3$ to $\mathrm{C} 4$ level, which was thought to represent cord contusion or edema. Based on the received

August 29, 2017

accepted

December 17, 2017
DOI https://doi.org/

10.1055/s-0038-1639341. ISSN 2193-6358.
๑) 2018 Georg Thieme Verlag KG
Stuttgart · New York

License terms

(®) $\Theta \circledast$ 


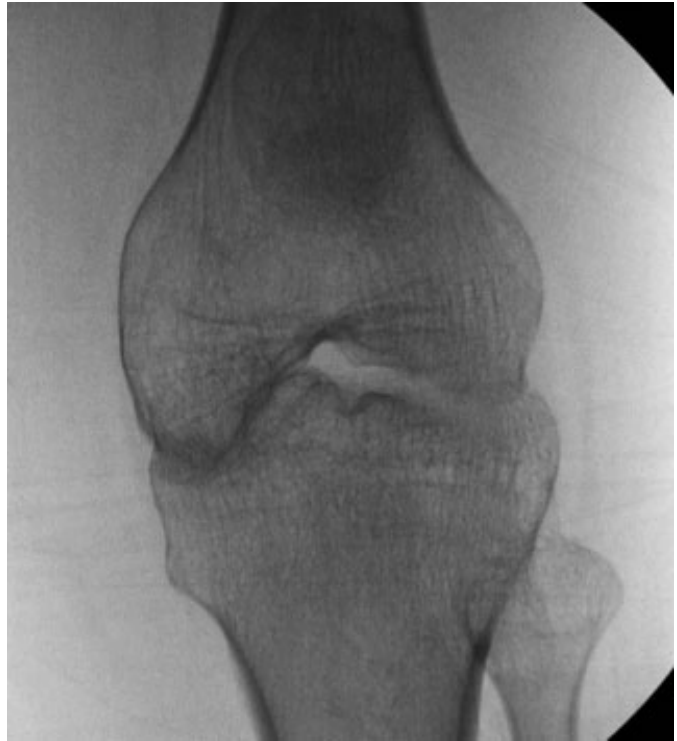

Fig. 1 Anteroposterior (AP) X-ray of the right knee. AP X-ray of the right knee demonstrating long-standing joint contractures and joint disease leading to a fused and nonfunctional knee joint.

imaging and clinical symptoms, a diagnosis of cervical spinal stenosis with myelopathy and right upper extremity radiculopathy was established. Risks and benefits of surgical decompression and fusion were discussed with the patient. A posterior cervical instrumented fusion from levels C3 to C6 was performed with a bilateral posterior cervical laminect- omy from C3 to C5. (- Fig. 3) Local laminectomy bone was utilized as morselized autograft with the addition of demineralized bone matrix to aid with fusion.

Postoperative MRI demonstrated proper alignment and decompression. (-Fig. 4) The patient experienced mild neck pain post-surgery; otherwise, the post-operative course was uneventful. Patient was kept in a cervical hard collar for 6 weeks postoperatively. At her 6-week follow-up, the patient reported improvement in strength and resolution of paresthesias in her right upper extremity. Hoffman's reflex could only be elicited on the right side now. At 6-month follow-up, patient returned to full strength in both her upper extremities and Hoffman's reflex was no longer present. Cervical X-rays demonstrated adequate fusion with maintenance of alignment and no evidence of hardware failure. She had returned to her full baseline mobility including selftransfer to wheelchair and was even learning to drive.

\section{Discussion}

AMC is an umbrella term that describes various congenital disorders in which two or more joint contractures at different locations in the body are present. ${ }^{1,3,5,7}$ The prevalence of arthrogryposis is estimated to be in $1 / 3000$ to $1 / 5000$ live births. ${ }^{7,8}$ Several pathologic causes for arthrogryposis exist, but $\sim 70 \%$ to $80 \%$ of cases have a neurogenic etiology. ${ }^{5}$ Contractures in arthrogryposis patients are most frequently present in shoulders, elbows, wrists, hips, knees, feet, and spine. ${ }^{6}$ Arthrogryposis patients may also experience other
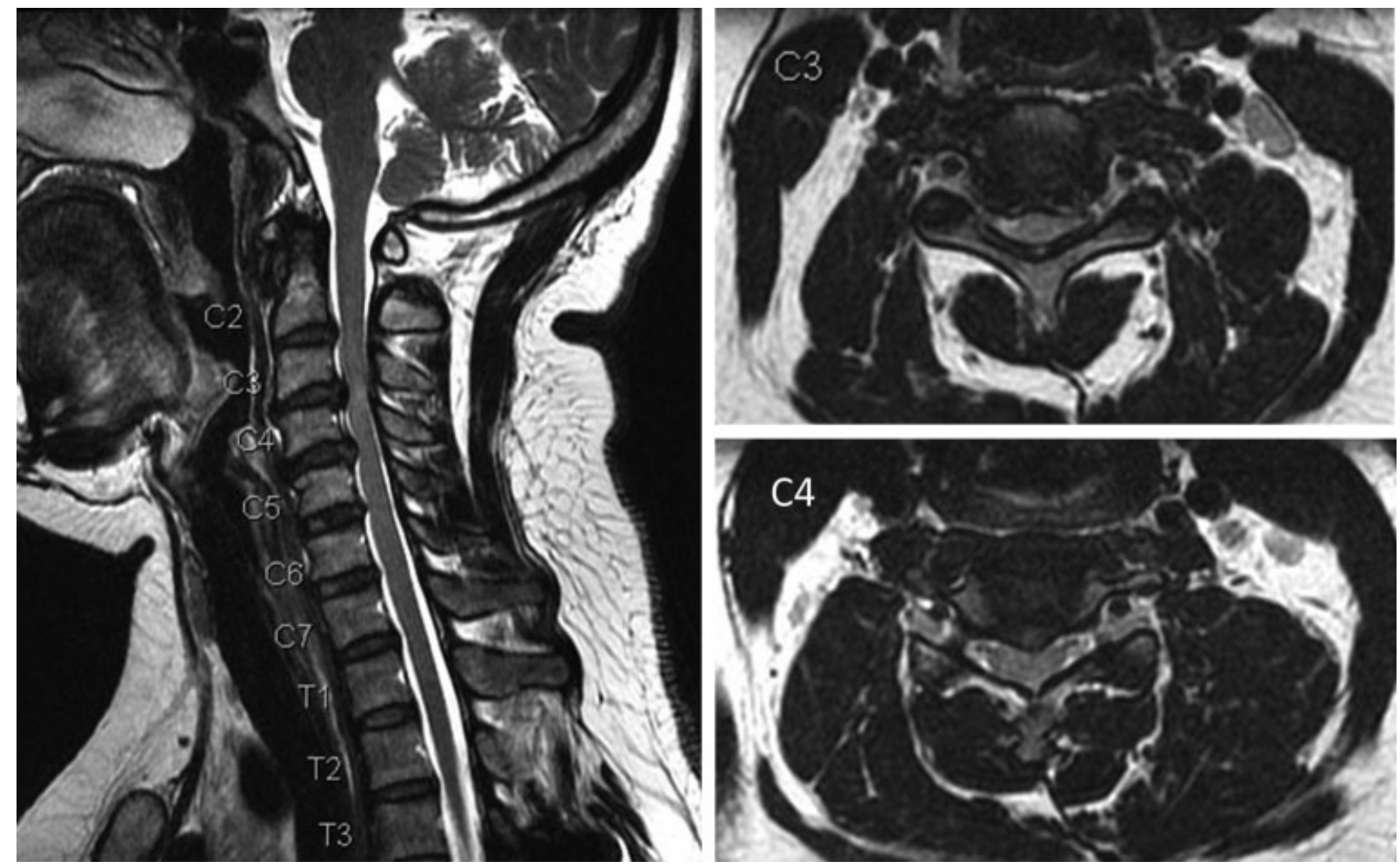

Fig. 2 Preoperative cervical magnetic resonance imaging (MRI). Preoperative cervical MRI demonstrating moderate-to-severe spinal stenosis from $\mathrm{C} 3$ to $\mathrm{C} 5$, and evidence of intramedullary spinal cord signal change at C3 to C4 level, which was thought to represent cord contusion or edema. 

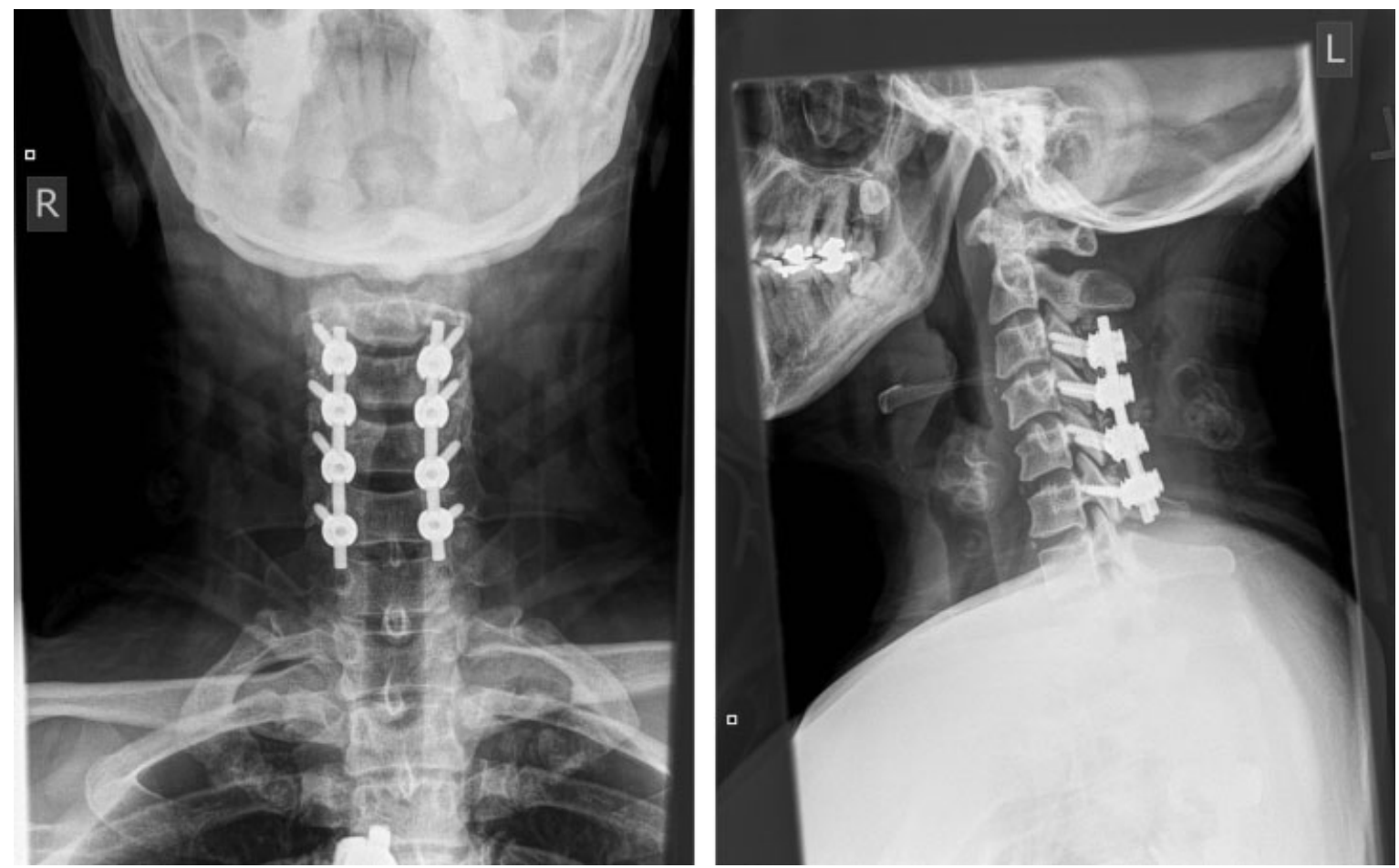

Fig. 3 Anteroposterior (AP) and lateral postoperative cervical X-rays. AP and lateral postoperative cervical X-rays demonstrating a posterior cervical instrumented fusion with good instrumentation placement.
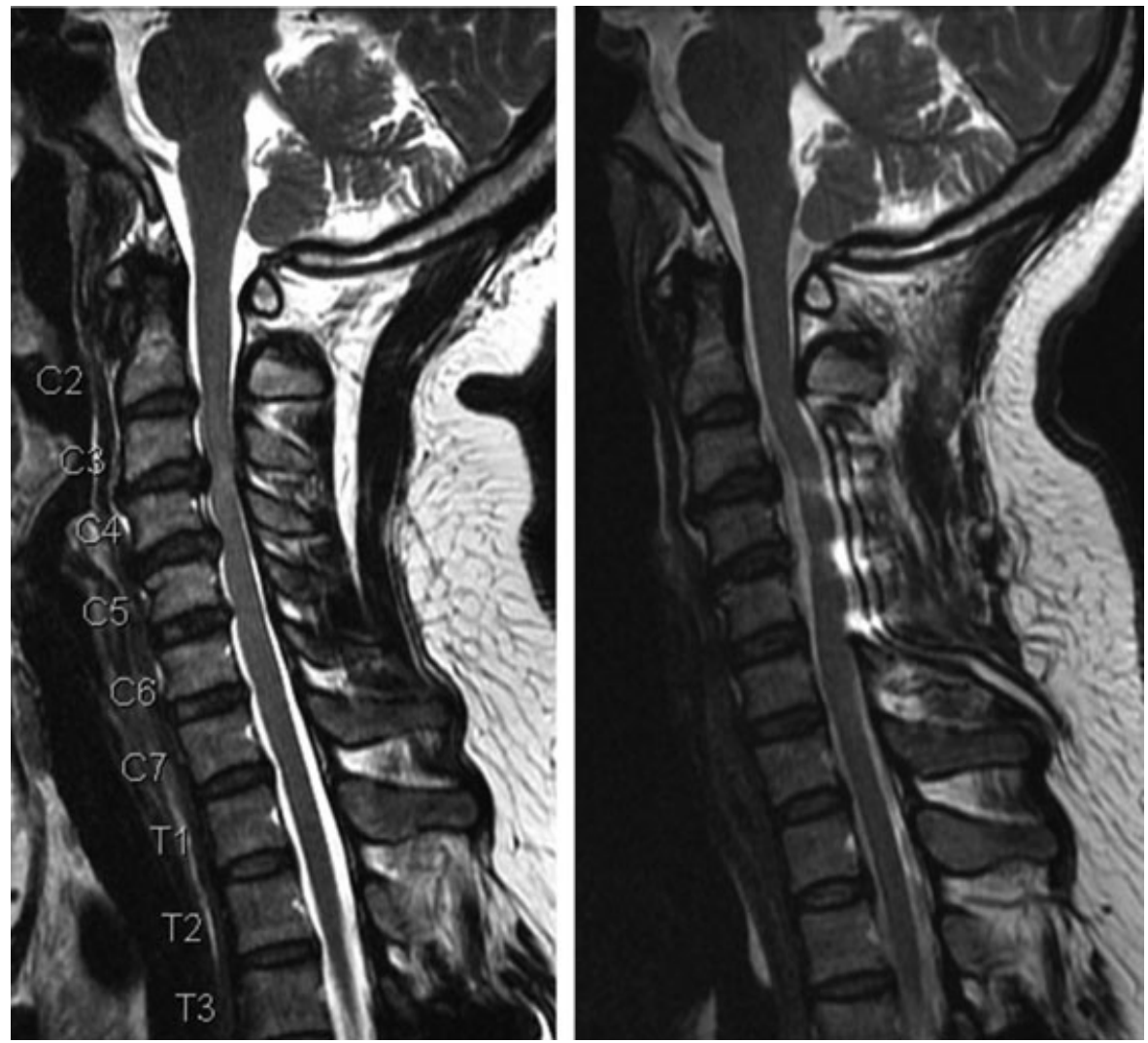

Fig. 4 Pre- and postoperative cervical magnetic resonance imaging (MRI). (Left) Preoperative cervical MRI demonstrating multilevel cervical stenosis with cord signal change. (Right) Postoperative cervical MRI demonstrating optimal cervical decompression and alignment. 
medical complications such as lung hypoplasia, respiratory problems, growth retardation, congenital heart defects, tracheoesophageal fistulas, and various other conditions. ${ }^{5}$

Management of arthrogryposis begins at an early age and requires the expertise of a multidisciplinary team of pediatricians, surgeons, physical therapists, physiatrists, nurses, and social workers. The goal of treatment is to maximize function and enable patients to live an independent life. ${ }^{9}$ Following their early corrective procedures, most arthrogryposis patients function well in adulthood, with a 20-year survival rate of $94 \%{ }^{7}$

Even though many patients with arthrogryposis function well into adulthood, in the present literature relatively few studies have documented the progression of the condition throughout adulthood. ${ }^{4}$ Among the few longitudinal studies, the focus is primarily on tracking independence, ambulation, and psychosocial functioning. ${ }^{4,6}$ Surgical management of these patients as adults seems to be understudied. Given the longevity of these patients, it is possible that accelerated degenerative changes in the spine due to this disorder can cause new neurologic symptoms that may require surgical treatment.

Involvement of the spine in arthrogryposis is usually in the form of scoliosis and ranges from a prevalence of $\sim 30 \%$ to $60 \%{ }^{1,4}$ To the author's knowledge, only two cases of spinal cord compression or spinal stenosis in adult patients with arthrogryposis have been reported. In a case report presented by Riemer and Steen, ${ }^{3}$ a 73-year-old woman with arthrogryposis developed lumbar stenosis which was successfully treated with a laminectomy and fixation. In the second case by Du et al, ${ }^{1}$ a 48 -year-old male with arthrogryposis and severe cervical (C3-C7) stenosis was surgically treated with an anterior cervical discectomy and fusion. Although the patient experienced temporary relief from the procedure and radiologic improvement was noted, the patient's initial symptoms of pain and weakness returned 1 year post-surgery.

Du et al's case demonstrates the challenges associated with the treatment of rare congenital disorders as it is often difficult to determine whether new symptoms are due to underlying congenital disease or of new etiology. Therefore, for each patient the surgeon must consider the underlying condition. In our case, it is unusual to see this degree of cervical stenosis with myelopathy in such a young patient and this was likely a sequela of her underlying arthrogryposis causing accelerated degenerative disc disease of the cervical spine. Indeed, she had clinical symptoms and radiologic evidence of cervical stenosis and spinal cord injury, suggesting that early degenerative cervical stenosis was the cause of her new neurologic symptoms.

After the decision to move forward with surgery is made, several perioperative issues must be considered in arthrogryposis patients to reduce complications. The presence of multiple contractures creates difficulty in obtaining venous access, endotracheal intubation, and proper positioning of patient. $^{7,8}$ Decreased muscle mass in these patients also influences the volume of anesthesia administered. Another potential risk factor that must be considered preoperatively in arthrogryposis patients is malignant hyperthermia. ${ }^{8}$ Consideration was given to both decompression alone and decompression with instrumented fusion options. Given the patient's young age, her risk of developing cervical kyphosis over time with decompression alone was significant. Additionally, as mentioned above, patients with arthrogryposis have globally decreased muscle mass. Such weak and under-developed posterior cervical musculature can also potentially contribute to higher rates of cervical kyphosis. Therefore, decompression and fusion are likely the optimal surgical strategy to avoid this complication later in life. In our patient, underdeveloped posterior cervical muscles were appreciated during exposure of the posterior cervical spine.

\section{Conclusion}

Arthrogryposis is a rare congenital disorder characterized by a constellation of symptoms including joint contractures and spine deformity that has been most well-studied in the pediatric population. We present a case of arthrogryposisrelated cervical stenosis in an adult that underwent posterior cervical decompression and instrumented fusion to achieve preservation of neurologic function. Adult-stage sequela of arthrogryposis can affect the spine and warrant unique surgical considerations to preserve neurologic function and alignment given the underlying congenital disease.

\section{References}

1 Du J, Aichmair A, Lykissas M, Girardi F. Cervical stenosis in a patient with arthrogryposis: case report. Evid Based Spine Care J 2014;5(01):57-62

2 Fletcher ND, Rathjen KE, Bush P, Ezaki M. Asymmetrical arthrogryposis of the upper extremity associated with congenital spine anomalies. J Pediatr Orthop 2010;30(08):936-941

3 Riemer G, Steen U. Amyoplasia: a case report of an old woman. Disabil Rehabil 2013;35(11):950-958

4 Fassier A, Wicart P, Dubousset J, Seringe R. Arthrogryposis multiplex congenita. Long-term follow-up from birth until skeletal maturity. J Child Orthop 2009;3(05):383-390

5 Kalampokas E, Kalampokas T, Sofoudis C, Deligeoroglou E, Botsis D. Diagnosing arthrogryposis multiplex congenita: a review. ISRN Obstet Gynecol 2012;2012:264918

6 Carlson WO, Speck GJ, Vicari V, Wenger DR. Arthrogryposis multiplex congenita. A long-term follow-up study. Clin Orthop Relat Res 1985;5(194):115-123

7 Bevan WP, Hall JG, Bamshad M, Staheli LT, Jaffe KM, Song K. Arthrogryposis multiplex congenita (amyoplasia): an orthopaedic perspective. J Pediatr Orthop 2007;27(05):594-600

8 Graydon AJ, Eastwood DM. Orthopaedic management of arthrogryposis multiplex congenita. European Surgical Orthopaedics and 2014:4627-4644

9 Thompson GH, Bilenker RM. Comprehensive management of arthrogryposis multiplex congenita. Clin Orthop Relat Res 1985;5(194):6-14 